\title{
Description of Anatomic Coordinate Systems and Rationale for Use in an Image-Guided Total Hip Replacement System
}

\author{
Constantinos Nikou, Branislav Jaramaz, Anthony M. DiGioia, and Timothy J. \\ Levison \\ Center for Medical Robotics and Computer-Assisted Surgery, UPMC Shadyside Hospital, \\ Pittsburgh, PA. \\ $\{$ [costa, branko, tony, levison]@cor.ssh.edu $\}$
}

\begin{abstract}
Lowering the risks of a surgical procedure is extremely important, especially for high-volume procedures such as total hip replacement. Significant work has been done to study total hip replacement procedures and provide the surgeon with techniques and tools to achieve better patient outcomes. Computer-assisted intervention allows surgeons to "close the loop" in medical research, allowing the surgeon to preoperatively plan, interoperatively navigate, and postoperatively analyze medical procedures, then use the results to repeat or improve the quality of future procedures. In order to expedite the cycle of planning, execution, and analysis amoung multiple research groups, standards for description, measurement, and procedure are necessary. In this work, the authors preset the coordinate systems used in their suite of computer-based tools for planning, executing, and evaluating the total hip replacement procedure. Rationales for the choices of each system are given along with experimental data which support the definitions.
\end{abstract}

\section{Introduction}

Total hip replacement (THR) is a very common and very successful procedure used to treat patient pain and immobility due to degenerative disease of the hip joint. Each year, about 250,000 hip replacement procedures are performed in the United States alone [1]. With such a large number of cases, even low percentages of failure are significant. Consistent failure of the prosthetic joint can require revision, which requires an extra surgical procedure, causing patient distress and incurring additional medical costs. Therefore, minimizing the risks of failure is critical.

Possible modes of failure include implant dislocation, excessive wear, loosening, and leg length discrepancy. All of these phenomena are affected by the positions of the prostheses in an artificial hip joint. Studies have shown a significantly lower risk of certain failure modes when the implants are placed within a "safe zone" [2]. However, patient-to-patient differences in anatomical structure and activity make general rules of implant placement a near impossibility.

Prior work done by the authors has attempted to lower the risk of failure by analyzing the THR procedure, recognizing its limitations, and providing a means to preoperatively plan [3], intraoperatively execute [4], and postoperatively evaluate [5] THR cases with a suite of computer-based tools known as HipNav. In this work, we describe the coordinate systems defined in HipNav and provide rationale for the choice of each. 


\section{Methods}

\subsection{Body Coordinate System}

There is an inherent body coordinate frame defined by the transverse, mid-sagittal, and coronal planes of the patient. This coordinate frame is used as a global orientation reference. The body $\mathrm{X}$-axis points to the left of the patient, perpendicular to the sagittal plane. The body $\mathrm{Y}$ - direction is normal to the transverse plane, pointing to the superior. All coordinate systems described in this work are right-handed coordinate systems, thereby defining the body Z-axis to lie normal to the coronal plane, pointing to the anterior (Figure 1).

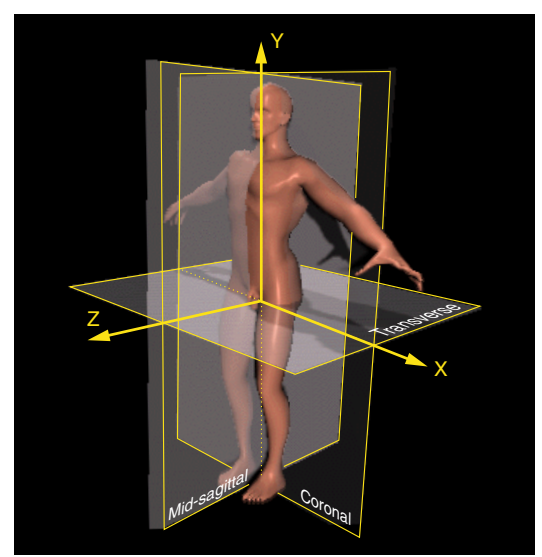

Fig. 1. Body coordinate system

\subsection{Pelvic Coordinate System}

The pelvic coordinate system is defined by four points on the pelvis: the maximally anterior left and right iliac spine points, and the maximally anterior left and right pubis symphysis points. The anterior spine points and the median of the pubis symphysis points define an anterior plane of the pelvis. This plane, along with the line between the iliac spine points, defines a coordinate system for the pelvis (Figure 2). The pelvic X-axis points to the patient's left, parallel with the line between the iliac spine points. The above. The pelvic Y-axis points to the superior, perpendicular to the other axes. The pelvic coordinate system is centered at the median of the iliac crest points, thereby making the definition rely wholly on the four landmark points.

It was hypothesized that the orientation of the APP in the average standing patient is roughly vertical, or parallel to the coronal (or here, the body XZ) plane. To test this hypothesis, lateral radiographs for 100 THR cases were analyzed. Rotation of the APP in the sagittal plane is easily visible in lateral radiographs. It was found that the standing position orientations showed a trend toward neutral alignment of the anterior pelvic plane, with a mean anterior rotation of 2 degrees relative to vertical (and a standard deviation of 8 degrees). We thereby can safely define "neutral standing position" (hereafter called simply "neutral position") of the pelvis to lie 


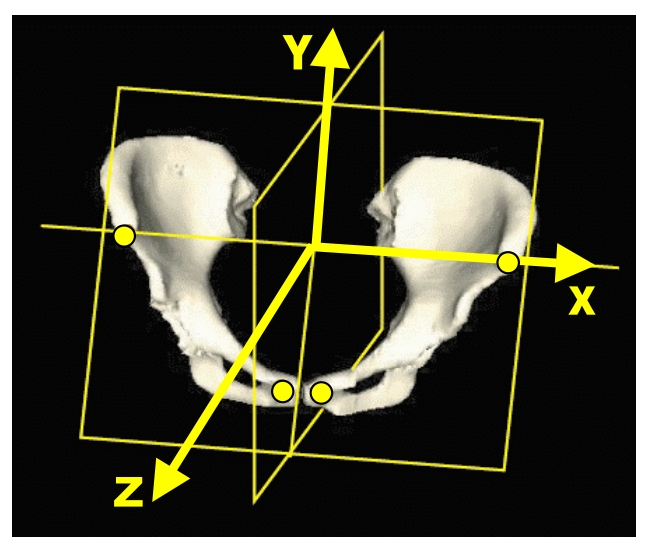

Fig. 2. Pelvic landmark points and the established coordinate frame

parallel with the body coordinate system. Any functional rotation (such as pelvic flexion) of the pelvis is defined relative to neutral position.

Measurements of APP orientation of sitting patients (mean posterior rotation of 35 degrees with a 14 degree standard deviation) were also done, reinforcing past studies $[4,6,7,8]$ stating the variability of functional pelvic position in patients both in and out of the operating room. This implies need of a definition of acetabular cup alignment that is independent of pelvic orientation.

\subsection{Acetabular Component Coordinate System}

The coordinate frame for the acetabular component is centered at the rotation center of the liner. The cup Z-axis points away from the liner opening and lies normal to the flat plane of the implant shell (Figure 3). If the implant liner is not axisymmetric (i.e. it has an extended lip), the $\mathrm{Y}$-axis marks the center of the extension. The orientation of the cup is commonly quantified using one of three paramaterizations: anatomic, operative and radiographic. The anatomic definition decribes the cup position in terms of cup abduction and anteversion. Neutral cup position orients the cup's Z-axis

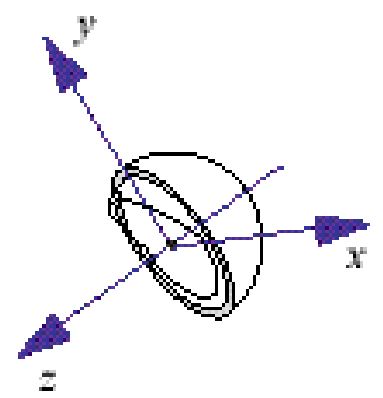

Fig. 3. Acetabular implant reference frame 
Table 1. Parameterization values for identical cup orientations. As the secondary value increases (anteversion or flexion), the parameter values become less consistent.

\begin{tabular}{|c|c|c|}
\hline $\begin{array}{c}\text { Abduction/ Radiographic } \\
\text { Version }\end{array}$ & Abduction/ Flexion & Abduction/ Anteversion \\
\hline $35 / 5$ & $35 / 6$ & $35 / 9$ \\
\hline $35 / 15$ & $34 / 18$ & $38 / 25$ \\
\hline $35 / 25$ & $31 / 30$ & $42 / 39$ \\
\hline $45 / 5$ & $45 / 7$ & $45 / 7$ \\
\hline $45 / 15$ & $43 / 21$ & $47 / 21$ \\
\hline $45 / 25$ & $40 / 33$ & $50 / 33$ \\
\hline $55 / 5$ & $55 / 9$ & $56 / 6$ \\
\hline $55 / 15$ & $52 / 25$ & $59 / 30$ \\
\hline $55 / 25$ & $48 / 39$ & 55 \\
\hline
\end{tabular}

pointing opposite the pelvic Y-axis, with the cup $\mathrm{Y}$-axis pointing laterally, parallel to the pelvic $\mathrm{X}$-axis. Abduction describes cup rotation from neutral around an axis through its center and parallel to the pelvic Z-axis (hereafter called pelvic Z-rotation). Anteversion in this case is a subsequent pelvic Y-rotation. The radiographic definition decribes the cup position in terms of cup abduction as above followed by cup anteversion, this time defined as cup rotation around the $\mathrm{Y}$-axis of the cup. This definition is often used in postoperative radiographic studies because this definition of anteversion is easily measured via the view of the cup in the X-ray image, and is therefore commonly used in postoperative studies. The operative definition decribes the cup position in terms of cup abduction followed by cup "flexion" (pelvic Xrotation). Traditional mechanical cup positioning guides are set to follow the abduction/flexion alignment and are therefore preset to achieve planned alignment in terms of this operative definition. All three systems are combined with a "twist" value that represents rotation of the implant about the cup axis. This is required when studying effects of non-axisymmetric cup liners on postoperative range of motion. These parameterizations are all relative to the pelvic coordinate system, which is important for describing cup position independent of the varying functional position of the pelvis, but they are not interchangeable. As we can see in Table 1, as anteversion (or flexion) increases the parameterization values diverge significantly.

\subsection{Femoral Coordinate System}

Four landmark points define the femoral coordinate system: the center of joint rotation, the posterior lesser trochanter, and the posterior femoral condyle points. The posterior lesser trochanter and condyle points define a plane. (Figure 4) The projection of the vector from the condylar midpoint to the joint center in this plane defines a vector parallel to the mechanical axis of the femur. The Z-axis of the femoral frame is normal to the defined plane. The $\mathrm{X}$-axis lies parallel to the plane and orthogonal to the mechanical axis. The Y-axis of the femoral frame is simply the resultant cross product. The femoral coordinate frame origin is located at the center of rotation for the femur. Therefore, because the Y-axis passes through the joint center (by definition) and, roughly, center of the knee joint, it lies along the mechanical axis of the femur. 


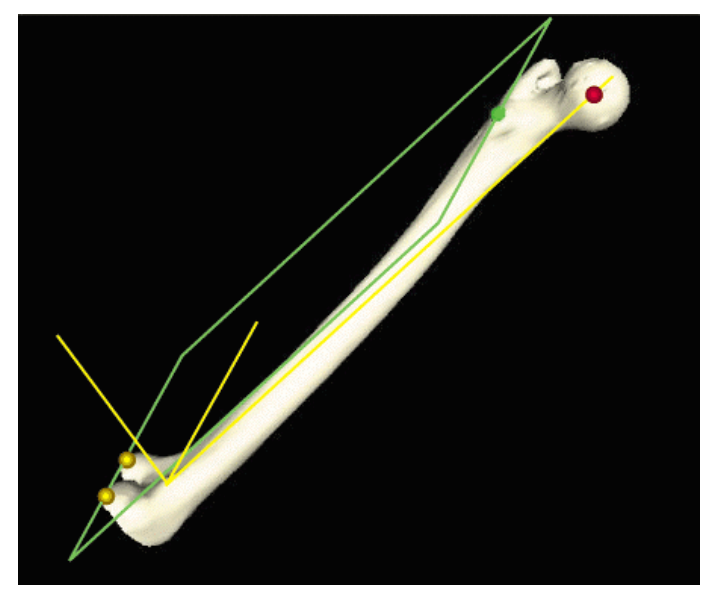

Fig. 4. Femoral landmark points which establish the femoral coordinate system

Note that because the femoral coordinate system is dependent on the center of rotation of the joint, changing the position of the joint center (which happens during THR) relative to the femur may change the orientation of the femoral frame. This change is most relevant in calculation of leg lengths and offsets, which are very important factors in determining proper component placement during THR. "Neutral position" of the femur is achieved when the coordinate frame of the femur lies parallel to the body coordinate frame. Any functional motion of the femur (discussed below in section 2.6) can be described as a rotation relative to the body coordinate system.

\subsection{Femoral Component Coordinate System}

The origin of the femoral implant neck coordinate frame is at the center of joint rotation, i.e. the center of the implant head. The orientation of the frame is parallel to the symmetry plane of the stem (Figure 5a). The Z-axis lies coincident with the axis of the implant neck, and the Y-axis is normal to the neck axis and in the symmetry

a)

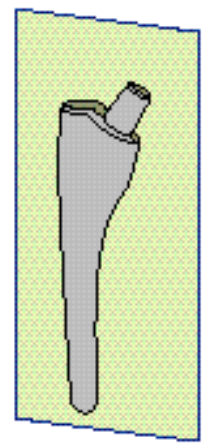

b)

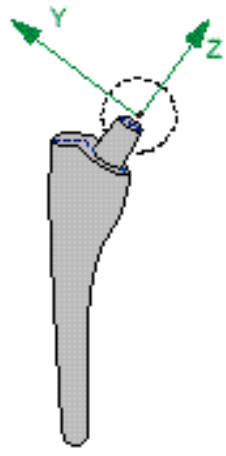

Fig. 5. a) Stem symmetry plane and b) neck coordinate frame 
plane (Figure 5b). Orientation of the implant neck relative to the can be described as a pair of rotations in the femoral coordinate frame: abduction and anteversion (or retroversion). These orientation parameters are analogous to their namesakes in acetabular component placement, with abduction as a femoral Z-rotation from vertical, followed by anteversion (a femoral Y-rotation). By using this parameterization, studies can be conducted on the effect "combined anteversion", or the difference of anteversion values for the femoral component neck and acetabular component placements, on postoperative range of motion [9].

\subsection{Function Leg Movement Definitions}

For purposes of simulation and analysis, the six functional movements of the femur (flexion, extension, abduction, adduction, internal rotation and external rotation) have been given precise geometrical definitions that follow the descriptions presented by Greene and Heckman [10]. Mathematically, these motions are pure rotations in the various coordinate frames described in previous sections. Any motion of the femur can be described as an ordered set of these rotations.

Flexion and extension describe rotation of the femur about the center of joint rotation parallel to the body YZ plane. Internal and external rotation are defined as rotations about the mechanical axis of the femur (femoral Y-axis). (Figure 6).
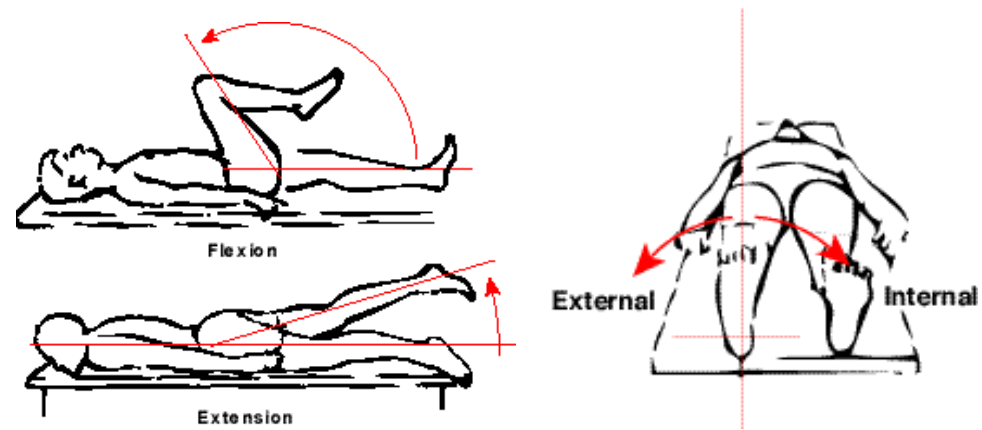

Fig. 6. Illustrations of femoral flexion, extension, internal and external rotation

(a)

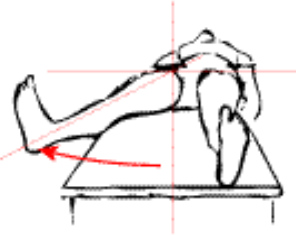

(b)

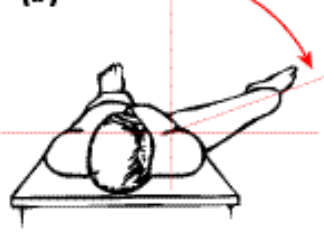

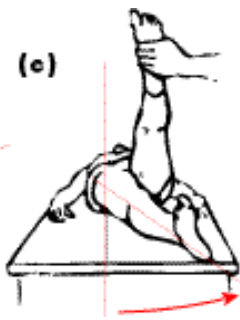

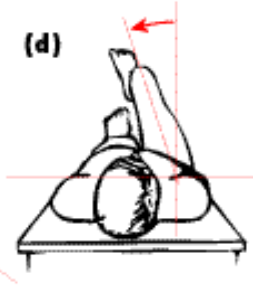

Fig. 7. Modes of abduction and adduction preceding and following flexion. (a) abduction, (b) abduction in flexion, (c) adduction, and (d) adduction in flexion 
The geometric definitions of femoral abduction and adduction depend on their occurrence relative to flexion or extension in the same motion. Abduction and adduction not taking place after flexion or extension describe rotations parallel to the body XY plane. When flexion (or extension) occurs before the ab/adduction, the movements are referred to as ab/adduction in flexion (or extension). In this case, they represent rotation parallel to the body XZ plane. (Figure 7).

\section{Discussion}

In this work, we have presented the anatomic and geometric definitions allowing quantification of results related to total hip replacement surgery. These definitions have been used in the HipNav computer-assisted THR system, and remain in use to allow repeatable results and ready application of information gained by the cases. It is important to rigorously define these coordinate systems and kinematic relationships when reporting results in order to eliminate confusion when comparing studies within and between research groups. In the future, standards for computer-assisted surgery in all disciplines will facilitate data integration allowing surgeons and engineers to improve medical practice and patient quality of life.

\section{References}

1. Healy, W. L. "The Economics of Total Hip Arthroplasty." From The Adult Hip. Callaghan, Rosenburgh, and Rubash, eds. 1998. Vol 2. pg. 845-852

2. Lewinnek, G. E., et al. Dislocation After Total Hip-Replacement Arthroplasties. Journal of Bone and Joint Surgery. Vol 60-A, No. 2, March 1978. pg. 217-220.

3. C. Nikou et al. POP: Preoperative Planning and Simulation Software for Total Hip Replacement Surgery. Second Int. Conf. in Med. Image Computing and ComputerAssisted Intervention (MICCAI'99). September 1999. pgs. 868-875

4. DiGioia et al. Image Guided Navigation System to Measure Intraoperatively Acetabular Implant Alignment. Clinical Orthopaedics and Related Research 355 (1998): 8-22

5. C. Nikou et al. CupAlign: Computer-Assisted Postoperative Radiographic Measurement of Acetabular Components Following Total Hip Arthroplasty. Second Int. Conf. in Med. Image Computing and Computer-Assisted Intervention (MICCAI'99). September 1999. pgs. $876-882$

6. Johnson, R. C. and Smidt, G. L. Measurement of Hip-Joint During Walking. J. of Bone and Joint Surgery. Vol 51-A:6, pgs. 1083-1094

7. Johnson, R. C. and Smidt, G. L. Hip Motion Measurements for Selected Activities of Daily Living. Clinical Orthopaedics and Related Research 72 (1970): pgs. 205-215

8. McCollum, D. E. and Gray, W. J.: Dislocation After Total Hip Arthroplasty (Causes and Prevention). Clinical Orthopaedics and Related Research 261 (1990). pg. 159-170

9. B. Jaramaz et al. Effect of Combined Acetabular/Femoral Implant Version on Hip Range of Motion. Trans. of the $45^{\text {th }}$ Annual Meeting of the Orthopaedic Research Society (ORS '99). Vol. 24. pg 926

10. Greene, W. B. and Heckman, J. D., eds. The Clinical Measurement of Joint Motion. 1994. pgs. 99-114 